Sharif University of Technology
Scientia Iranica
SCIENTIA

\title{
Fluid-structure interaction analysis of a piezoelectric flexible plate in a cavity filled with fluid
}

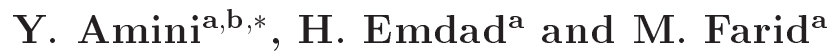 \\ a. School of Mechanical Engineering, Shiraz University, Shiraz, Iran. \\ b. Department of Mechanical Engineering, Persian Gulf University, Bushehr, Iran. \\ Received 8 January 2013; received in revised form 6 September 2014; accepted 16 June 2015
}

\author{
KEYWORDS \\ Fluid-structure \\ interaction; \\ Piezoelectric \\ actuators; \\ Smart materials; \\ Large structural \\ deformations.
}

\begin{abstract}
This study presents a numerical analysis of fluid-structure interaction, the structure of which is a flexible piezoelectric material. Piezoelectric materials are widely used in aero-elasticity and turbomachinery fields for vibration, flutter, and noise control. In this work, a FSI benchmark is revised to contain the piezoelectric materials. The influence of piezoelectricity on the oscillation of the structure and fluid flow is considered. For validation, two benchmark problems are solved and the results of the present code are compared with those of previous work. Current results show that the piezoelectric behavior of a plate significantly influences the oscillation of the plate and the fluid flow properties.

(C) 2016 Sharif University of Technology. All rights reserved.
\end{abstract}

\section{Introduction}

Fluid-Structure Interaction (FSI) plays an important role in many engineering applications, such as suspension bridges, aero-elasticity and flutter, bio-mechanics, oil platforms, power lines, aircraft wings, and turbomachinary.

There are two main computational categories for the modeling of FSI problems; the monolithic approach $[1,2]$ and the partitioned (segregated) approach $[3,4]$. In the monolithic approach, the same primitive variables (usually velocity and pressure) are used for both the fluid and solid domains; therefore, the governing equations of the fluid and structure are solved simultaneously with a single solver. On the other hand, in the partitioned approach, the fluid flow and the movement of the structure are solved separately by two distinct solvers. Since, in the

\footnotetext{
*. Corresponding author. Tel.: +98 7136133011 ; Fax: +98 7136473511

E-mail addresses: aminiyaser@shirazu.ac.ir, and aminiyasser@pgu.ac.ir (Y.Amini); hemdad@shirazu.ac.ir (H. Emdad); farid@shirazu.ac.ir (M. Farid)
}

monolithic approach, the nonlinear resulting equations arising from the coupled discretisation of the fluid and the structure are solved as a whole, use of a coupling algorithm is not required, and is the main advantage of this approach. However, the monolithic solvers are computationally expensive and sometimes the matrix of the resulting algebraic system can be ill-conditioned, leading to difficulties in solving these equations. The advantage of the partitioned approach is that it can precisely solve flow and structure equations by using different efficient techniques for each of them. Also, this approach uses separate meshes for the structure and the fluid, which often require different mesh resolutions. On the other hand, in the partitioned simulations, development of stable and accurate coupling algorithms is required.

Piezoelectric materials have been extensively used as sensor and actuator devices in aeroelasticity and turbomachinery fields for vibration, noise, buffet-induced vibration and flutter controls. In these problems, structural vibrations are produced as the result of interaction between the fluid flow and the structure. Therefore, all these problems are a type of FSI problem. 
Based on the authors' knowled ge, regarding previous studies in FSI problems with orthotropic piezoelectric materials, only piezoelectric and structural governing equations are solved, and the fluid flow is considered a pressure load [5] or calculated by the piston theory [69]. Therefore, fluid structure interaction was not taken into account.

Bolzmacher et al. [10] introduced a novel micromechanical amplification unit for increasing the stroke of piezoelectric micro-actuators. In their study, only the equation of structure was numerically solved. In order to obtain a better estimate for the actuator performance and to optimize its design, they proposed that the fluid-structure interaction between the piezoelectric actuator and the airflow should be considered in the simulations.

Recently, piezoelectric materials have been used as energy harvesters, which convert aero-elastic vibrations to electric energy [5,11-16]. Although these problems are of a FSI type, they were investigated by experimental analysis or a predefined vibration was assumed in numeric simulation. A full FSI analysis has not yet been undertaken.

By development of FSI codes and software, it is possible to solve these problems without simplification in the fluid flow and investigate them as a FSI problem. In this work, an FSI benchmark is revised to contain the piezoelectric materials. The influence of different electric potentials on deformation of the structure and the fluid flow is considered.

\section{Governing equations}

\subsection{Fluid equations}

The governing equations of the fluid flow are the incompressible Navier-Stokes equations. Since, in the Arbitrary Lagrangian Eulerian method (ALE) formulation, the mesh nodes of the fluid domain could be in motion, Navier-Stokes equations on a moving mesh should be used in the following form $[17,18]$ :

$$
\begin{aligned}
& \nabla \cdot v=0, \\
& \rho \frac{\partial v}{\partial t}+\rho(v-\hat{v}) \nabla \cdot v=-\nabla p+\mu \nabla^{2} v,
\end{aligned}
$$

where $\rho, v, p, \mu$ and $t$ are density, velocity, pressure, viscosity, and time, respectively, and $\hat{v}$ represent the mesh node velocity.

\subsection{Piezoelectric equations}

Some materials have the ability to convert mechanical energy into electrical energy and vice versa. This property is known as piezoelectricity. The direct piezoelectric effect is known as producing voltage by a material when it is subjected to stress. The inverse piezoelectric effect is that the crystal deforms when a voltage difference is applied across it.
The momentum equation for a solid material is:

$$
\rho \frac{\partial^{2} u}{\partial t^{2}}=\nabla . T
$$

where $T$ is the stress vector and $u$ is the displacement of the solid material.

The electric displacement (electric flux density) vector, $D_{i}$, satisfies Gauss law as:

$$
D_{i, i}=0 \text {. }
$$

The constitutive relations for a linear piezoelectric material are defined as:

$$
\begin{aligned}
& T_{i j}=c_{i j k l}^{E} S_{k l}-e_{k i j} E_{k}, \\
& D_{i}=e_{i k l} S_{k l}+\varepsilon_{i j}^{S} E_{k},
\end{aligned}
$$

where $S$ is the strain vector, $E$ is the electric field vector, $c$ is the elasticity matrix, $e$ is the piezoelectric stress matrix, and $\varepsilon$ is the dielectric matrix (evaluated at constant mechanical strain), respectively.

By substituting these constitutive relations in the momentum equations and Gauss law and using the following equations:

$$
\begin{aligned}
& E_{i}=-\varphi_{, i}, \\
& S_{i j}=\frac{1}{2}\left(u_{i, j}+u_{j, i}\right),
\end{aligned}
$$

the final set of partial differential equations with mechanical displacement and electric potential as primary dependent variables is obtained as:

$$
\begin{aligned}
& \rho \ddot{u}=\nabla \cdot[c: \nabla u]+\nabla \cdot[e \cdot \nabla \varphi], \\
& \nabla \cdot[e: \nabla u]-\nabla \cdot[\varepsilon \cdot \nabla \varphi]=0,
\end{aligned}
$$

which can be shown in the following index notation for constant material properties:

$$
\begin{aligned}
& \rho \ddot{u}_{i}=c_{i j k l}^{E} u_{k, l j}+e_{k i j} \varphi_{, k j}, \\
& e_{k i j} u_{i, k j}-\varepsilon_{k j}^{S} \varphi_{, k j}=0 .
\end{aligned}
$$

By using finite element discretization, the following system of coupled equations are derived for each element [9]:

$$
\begin{aligned}
{\left[\begin{array}{cc}
{[M]} & {[0]} \\
{[0]} & {[0]}
\end{array}\right]\left\{\begin{array}{l}
\{\ddot{u}\} \\
\{\ddot{\varphi}\}
\end{array}\right\}+\left[\begin{array}{ll}
{[C]} & {[0]} \\
{[0]} & {[0]}
\end{array}\right]\left\{\begin{array}{l}
\{\dot{u}\} \\
\{\dot{\varphi}\}
\end{array}\right\} } \\
+\left[\begin{array}{cc}
{[K]} & {\left[K^{z}\right]} \\
{\left[K^{z}\right]^{T}} & {\left[K^{d}\right]}
\end{array}\right]\left\{\begin{array}{l}
\{u\} \\
\{\varphi\}
\end{array}\right\}=\left\{\begin{array}{l}
\{F\} \\
\{L\}
\end{array}\right\},
\end{aligned}
$$

where $[M],[C]$, and $[K]$ are the element mass matrix, the element structural damping matrix, and the element stiffness matrix, respectively; $K^{z}$ is the piezoelectric coupling matrix, $K^{d}$ is the element dielectric permittivity coefficient matrix, $\{u\}$ is the displacement vector and $\{\varphi\},\{F\}$, and $\{L\}$ are the electric potential (voltage), structural and electrical load vectors, respectively. 


\subsection{Fluid-structure coupling and mesh motion} Usually, Lagrangian formulation, where each individual node of the computational mesh moves with the associated material particle during the motion, is used in solid mechanics. The Lagrangian description permits an easy tracking of free surfaces and interfaces among different materials. However, it requires remeshing processes for large distortions of the computational mesh.

Flow solvers often use an Eulerian description, in which the computational mesh is fixed and the fluid moves relative to the grid, and large distortions in the fluid motion are handled by this description. In the geometrically fixed fluid domain, this approach works well, but, difficulties arise when the boundaries of the fluid domain change. Fluid structure interaction is the most well-known example for moving boundaries in the fluid domain. In FSI problems, deformation in the solid material moves the fluid mesh points at the fluid-structure interface.

Various techniques have been proposed for simulation of the moving boundaries in the fluid domain. ALE is the most popular method employed for tracking fluid moving boundaries $[17,19]$. In an ALE method, some parts of the mesh can be moved with the associated material particles, such as the Lagrangian approach. Other parts of the mesh are fixed similar to the Eulerian approach. Although this method has low computational cost and it is accurate, for large deformations or movement of solid material, fluid elements tend to become ill-shaped and the solution accuracy reduces. Some alternatives to the ALE method are the immersed boundary method [20,21], fictitious domain method [22], and the mortar finite element method [23]. In contrast to the ALE technique, other methods use fixed fluid meshes, and, hence, can simulate large deformation of solid material without mesh folding in the fluid domain. However, since the ALE method captures the fluid-structure interface accurately, it has the ability to maintain high-quality meshes near the structure's interface. In the current study, a partitioned FSI solver with ALE formulation for the flow solver is used.

At the interface of the fluid and structure domains, interaction is considered by transferring the fluid flow stress to the structure and the structural displacements to the fluid flow. Also, it is assumed that the fluid always remains in contact with the structure. Therefore, the compatibility of the displacement and no-slip condition requires that:

$$
\begin{aligned}
& T^{S} . n=T^{F} . n, \\
& u^{S}=u^{F} .
\end{aligned}
$$

In the ALE approach, the fluid internal mesh should be deformed in response to the fluid-structure interface displacements. However, the deformation of the fluid domain is unknown and it should be found in terms of structural deformation. In the present work, the Laplacian operator is used for mesh motion in the fluid domain. In this method, mesh velocity, $\hat{v}$, is computed by solving the Laplacian equation as:

$$
\nabla \cdot(\gamma \nabla \hat{v})=0
$$

where $\gamma$ is the diffusion coefficient. The boundary conditions for this equation are:

$$
\hat{v}=\left\{\begin{array}{ll}
\frac{d u}{d t} & \text { for fluid solid interface boundary } \\
0 & \text { for other boundaries }
\end{array}\right\} .
$$

\section{Results and discussion}

Validation of the present work is achieved by analyzing two benchmark problems. The first is a piezoelectric problem and the other a fluid structural interaction problem. The results show that in the current study, the piezoelectric problem and the FSI problem are solved correctly. In order to investigate the effect of piezoelectricity on the fluid flow and deformation of the structure, the elastic material in the FSI benchmark is replaced by a piezoelectric material.

\subsection{Piezoelectric bimorph actuator plate}

The geometry of a piezoelectric bimorph plate is shown in Figure 1.

In this case, an electric potential equal to 50 voltages is applied to the bottom and top faces of the plate, and the intermediate surface is set to zero voltage. The length $(L)$, width $(l)$ and thickness of this rectangular plate are $25 \mathrm{~mm}, 12.5 \mathrm{~mm}$, and $2.5 \mathrm{~mm}$, respectively. Boundaries are considered to be simply supported. Also, its material constants are [24]:

$$
c=\left[\begin{array}{cccccc}
139 & 77.8 & 74.3 & 0 & 0 & 0 \\
77.8 & 139 & 74.3 & 0 & 0 & 0 \\
74.3 & 74.3 & 115 & 0 & 0 & 0 \\
0 & 0 & 0 & 25.6 & 0 & 0 \\
0 & 0 & 0 & 0 & 25.6 & 0 \\
0 & 0 & 0 & 0 & 0 & 30.6
\end{array}\right]
$$

GPa,

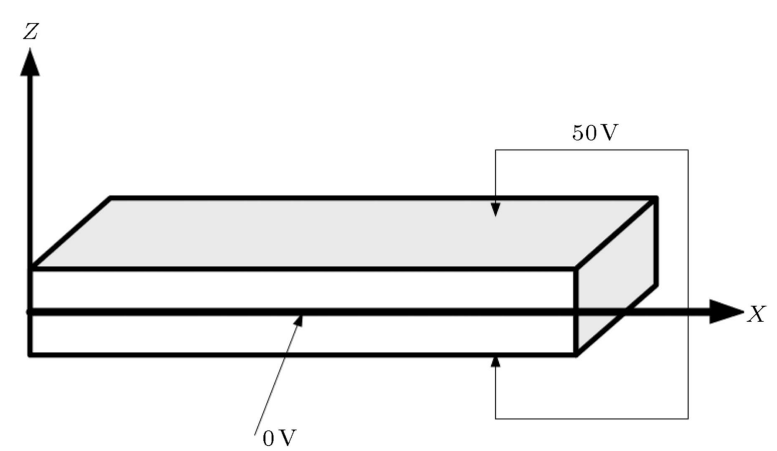

Figure 1. Piezoelectric bimorph geometry. 


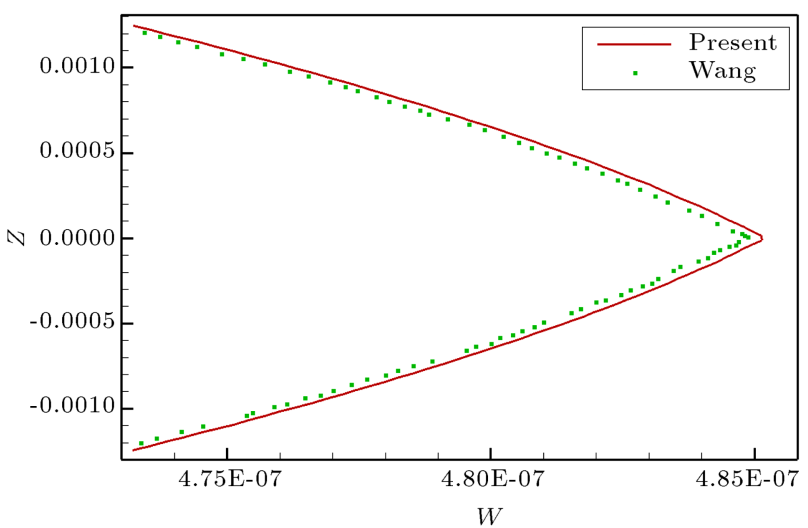

Figure 2. Through-the-thickness variation of the deflection, $W$, at the plate center.

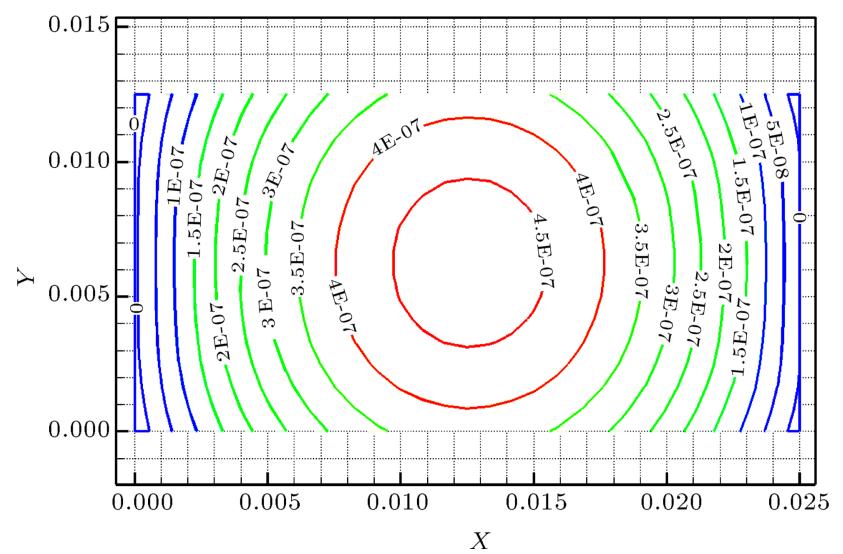

Figure 3. Contour of the through-the-thickness variation of the deflection, $W$, at the center of plate.

$$
\begin{aligned}
e & =\left[\begin{array}{ccc}
0 & 0 & -5.2 \\
0 & 0 & -5.2 \\
0 & 0 & 15.1 \\
0 & 12.7 & 0 \\
12.7 & 0 & 0 \\
0 & 0 & 0
\end{array}\right] \quad \frac{\mathrm{C}}{\mathrm{m}^{2}}, \\
\varepsilon & =\left[\begin{array}{ccc}
13.06 e-9 & 0 & 0 \\
0 & 13.06 e-9 & 0 \\
0 & 0 & 15.1 e-9
\end{array}\right] \quad \frac{\mathrm{F}}{\mathrm{m}} .
\end{aligned}
$$

In Figure 2, the through-the-thickness variation of deflection, $W$, at the plate center is shown and the results are compared with those of Wang [24]. From this figure, it is clear that in the present analysis, modeling of the piezoelectric material is done properly. Figure 3 shows the contours of deflection, $W$.

\subsection{Oscillation of a vertical flexible plate in a cavity}

The FSI part of the current work is validated by analyzing the oscillations of a vertical flexible plate in a cavity filled with fluid. The geometry of this case is shown in Figure 4.

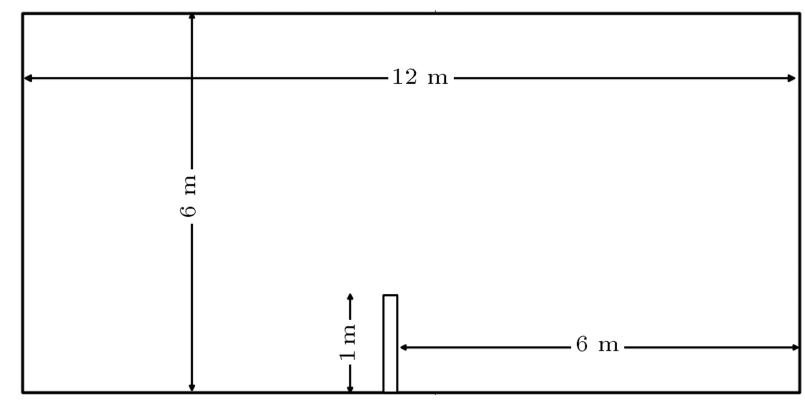

Figure 4. The geometry of FSI benchmark problem.

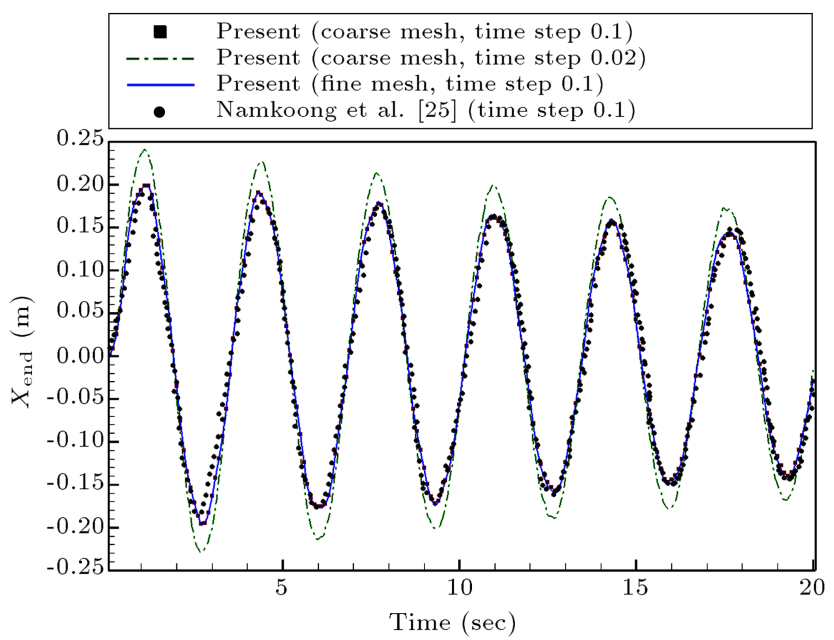

Figure 5. Comparison between the current study and previous results.

The length, thickness and width of the plate are $1.0 \mathrm{~m}, 0.06 \mathrm{~m}$ and $0.4 \mathrm{~m}$, respectively. Young's modulus of elasticity $(E)$ and the density of the plate $\left(\rho_{s}\right)$ are $2.5 \times 10^{6} \mathrm{~Pa}$ and $2550 \mathrm{~kg} / \mathrm{m}^{3}$, respectively.

The fluid has the following material properties: fluid density, $\rho_{f}=1 \mathrm{~kg} / \mathrm{m}^{3}$, and dynamic viscosity of the fluid, $\mu_{f}=0.2 \mathrm{~kg} / \mathrm{m}^{3}$. The plate is excited with a uniformly distributed load of $30 \mathrm{~N} / \mathrm{m}$ within the first 0.5 sec. Namkoong et al. [25] and Gluck et al. [26] solved this problem with a time step equal to 0.1 sec.

In Figure 5, the horizontal displacement of the free end of the plate obtained by Namkoong et al. and the current solver are compared. From this figure, it is evident that when the time step is chosen the same as that in the work of Namkoong et al., the results of the current study agree with those obtained by them. But, this time step is very large for this problem. By reducing the time step, the results change and we propose that for later studies, results should be compared with a time step of $0.02 \mathrm{sec}$ or less.

\subsection{Oscillation of a vertical piezoelectric plate in a cavity}

For investigation of the effect of piezoelectricity on structural deformation and the flow field, the elastic plate in the previous FSI problem is replaced by a plate 


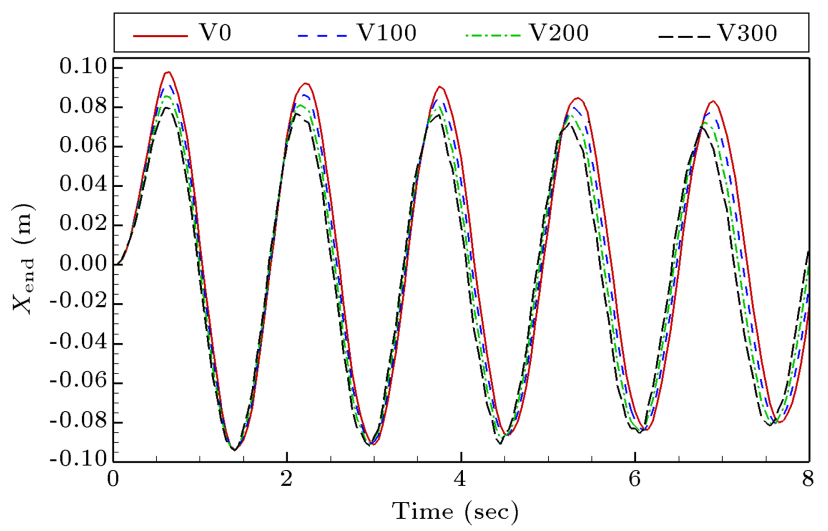

Figure 6. Horizontal displacement of the free end of the piezoelectric plate for different voltages.

made of piezoelectric material. The problem geometry, length, width and thickness of the piezoelectric plate and the fluid parameters are chosen similar to those of the preceding case. The material constants of the piezoelectric plate are considered to be:

$$
\begin{aligned}
& c=\left[\begin{array}{cccccc}
139 & 77.8 & 74.3 & 0 & 0 & 0 \\
77.8 & 139 & 74.3 & 0 & 0 & 0 \\
74.3 & 74.3 & 115 & 0 & 0 & 0 \\
0 & 0 & 0 & 25.6 & 0 & 0 \\
0 & 0 & 0 & 0 & 25.6 & 0 \\
0 & 0 & 0 & 0 & 0 & 30.6
\end{array}\right] \quad \mathrm{MPa} \\
& e=\left[\begin{array}{ccc}
0 & 0 & -5.2 \\
0 & 0 & -5.2 \\
0 & 0 & 15.1 \\
0 & 12.7 & 0 \\
12.7 & 0 & 0 \\
0 & 0 & 0
\end{array}\right] \quad \frac{\mathrm{C}}{\mathrm{m}^{2}} \\
& \varepsilon=\left[\begin{array}{ccc}
13.06 e-9 & 0 & 0 \\
0 & 13.06 e-9 & 0 \\
0 & 0 & 15.1 e-9
\end{array}\right] \quad \frac{\mathrm{F}}{\mathrm{m}}
\end{aligned}
$$

In this problem, the voltage at the right face of the plate is set to zero, but, various voltages are applied at the left face of the plate.

Figure 6 presents the horizontal displacement of the free end of the piezoelectric plate for different voltages $(0,100,200$ and 300 volts). By applying these voltages to the piezoelectric plate, the maximum deflection of the plate in the positive $x$ direction is reduced, but, no significant effect on maximum deflection in the negative $x$ direction is seen. The reason for this phenomenon is that the applied voltages tend to deform the plate in the negative direction of $x$.

For electric potential equal to $200 \mathrm{~V}$, the deformations of the left face of the plate are shown for different times in Figure 7 . This figure reveals that this high voltage does not activate the higher mode shapes in the plate.

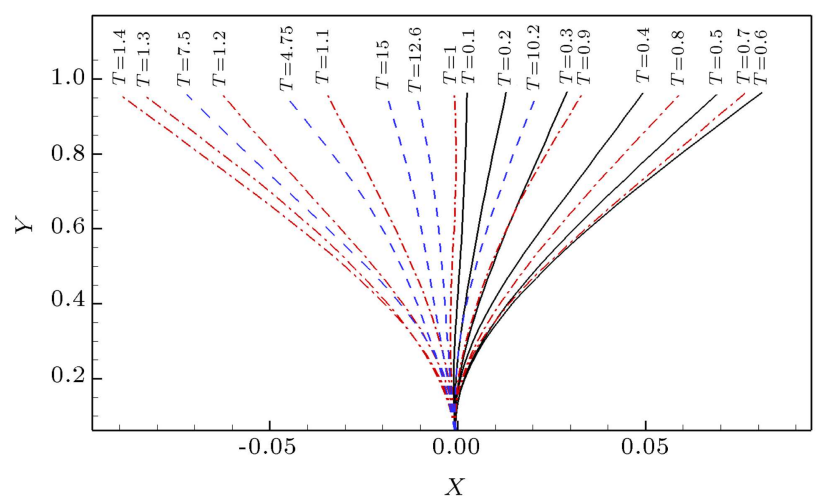

Figure 7. Deformations of left face of the plate at electric potential equal to $200 \mathrm{~V}$ for different times.

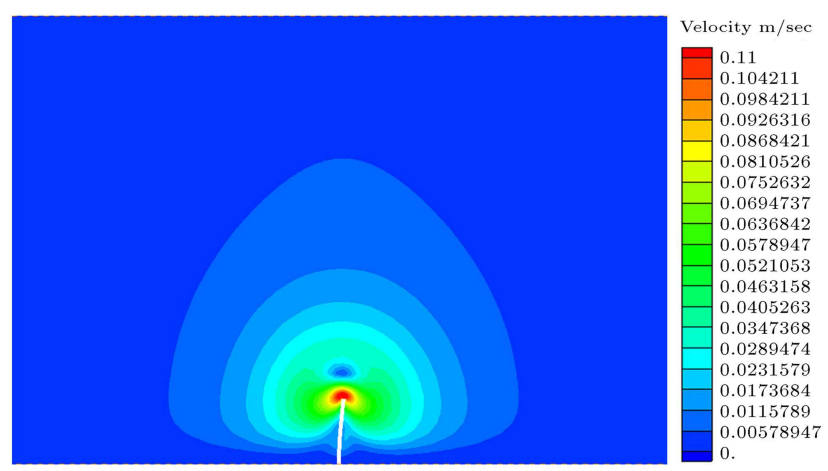

Figure 8. Velocity contours for electric potential of $200 \mathrm{~V}$ and time of $0.6 \mathrm{sec}$.

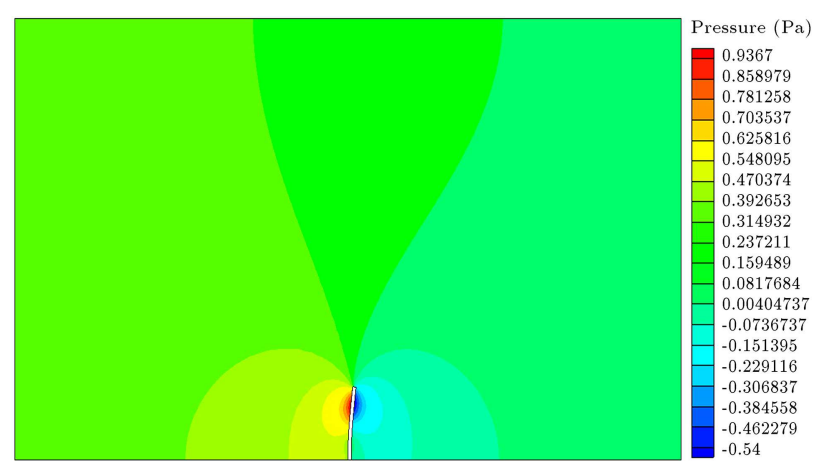

Figure 9. Pressure contours for electric potential of $200 \mathrm{~V}$ and time of $0.6 \mathrm{sec}$.

Figures 8 and 9 show the velocity and pressure contours for electric potential of $200 \mathrm{~V}$ and time of $0.6 \mathrm{sec}$, respectively. As shown in Figure 8, at the top of the plate, the induced velocity in the fluid domain has its maximum value.

\section{Conclusion}

In the present work, an FSI benchmark problem was revised to contain the orthotropic piezoelectric material. In this problem, different electric voltages were applied to the piezoelectric material and the vibration of this plate at different times was considered. It 
was observed that by increasing the electric potential from zero, maximum displacement in the positive $x$ direction is reduced. However, the influence of the electric potential on the plate deflection is decreased by increasing the electric potential. Therefore, the piezoelectric actuator suppresses the vibration of the plate, which is caused by fluid flow.

\section{References}

1. Blom, F.J. "A monolithical fluid-structure interaction algorithm applied to the piston problem", Comput. Methods Appl. Mech. Eng., 167(3-4), pp. 369-391 (1998).

2. Dunne, T., Rannacher, R. and Richter, T. "Numerical simulation of fluid-structure interaction based on monolithic variational formulations", Numerical Fluid Structure Interaction, Springer (2010).

3. Piperno, S. and Farhat, C. "Partitioned procedures for the transient solution of coupled aeroelastic problems Part II: energy transfer analysis and three-dimensional applications", Comput. Methods Appl. Mech. Eng., 190(24-25), pp. 3147-3170 (2001).

4. Piperno, S., Farhat, C. and Larrouturou, B. "Partitioned procedures for the transient solution of coupled aroelastic problems. Part I: Model problem, theory and two-dimensional application", Comput. Methods Appl. Mech. Eng., 124(1-2), pp. 79-112 (1995).

5. Dunnmon, J.A., Stanton, S.C., Mann, B.P. and Dowell, E.H. "Power extraction from aeroelastic limit cycle oscillations", J Fluids Struct., 27(8), pp. 1182-1198 (2011).

6. Moon, S.H. "Finite element analysis and design of control system with feedback output using piezoelectric sensor/actuator for panel flutter suppression", Finite Elements in Analysis and Design, 42(12), pp. 10711078 (2006).

7. Kouchakzadeh, M.A., Rasekh, M. and Haddadpour, H. "Panel flutter analysis of general laminated composite plates", Compos. Struct., 92(12), pp. 2906-2915 (2010).

8. Li, F.-M. "Active aeroelastic flutter suppression of a supersonic plate with piezoelectric material", Int. J. Eng. Sci., 51(0), pp. 190-203 (2012).

9. Allik, H. and Hughes, T.J.R. "Finite element method for piezoelectric vibration", Int. J. Numer. Methods Eng., 2(2), pp. 151-157 (1970).

10. Bolzmacher, C., Bauer, K., Schmid, U., Hafez, M. and Seidel, H. "Displacement amplification of piezoelectric microactuators with a micromachined leverage unit", Sensors and Actuators A: Physical, 157(1), pp. 61-67 (2010).

11. Erturk, A., Tarazaga, P.A., Farmer, J.R. and Inman, D.J. "Effect of strain nodes and electrode configuration on piezoelectric energy harvesting from cantilevered beams", Journal of Vibration and Acoustics, 131(1), pp. 011010-011011 (2009).
12. Erturk, A., Tarazaga, P.A., Farmer, J.R. and Inman, D.J. "Effect of strain nodes and electrode configuration on piezoelectric energy harvesting from cantilevered beams", Journal of Vibration and Acoustics, 131(1), p. 011010 (2009).

13. Lee, S. and Youn, B.D. "A design and experimental verification methodology for an energy harvester skin structure", Smart Materials and Structures, 20(5), pp. 057001 (2011).

14. Singh, K., Michelin, S. and de Langre, E. "Energy harvesting from axial fluid-elastic instabilities of a cylinder", J. Fluids Struct., 30(0), pp. 159-172 (2012).

15. Soobum, L. and Byeng Dong, Y. "A new energy harvesting design concept: Multimodal energy harvesting skin", In 13th AIAA/ISSMO Multidisciplinary Analysis Optimization Conference, American Institute of Aeronautics and Astronautics (2010).

16. Soobum, L. and Youn, B.D. "A new piezoelectric energy harvesting design concept: multimodal energy harvesting skin", IEEE Transactions on Ultrasonics, Ferroelectrics and Frequency Control, 58(3), pp. 629645 (2011).

17. Donea, J., Huerta, A., Ponthot, J.P. and RodríguezFerran, A. "Arbitrary Lagrangian-Eulerian methods", In Encyclopedia of Computational Mechanics, John Wiley \& Sons, Ltd (2004).

18. Wang, X.S., Fundamentals of Fluid-Solid Interactions, Elsevier, Amsterdam, London (2008).

19. Donea, J., Giuliani, S. and Halleux, J.P. "An arbitrary Lagrangian-Eulerian finite element method for transient dynamic fluid-structure interactions", Comput. Methods Appl. Mech. Eng., 33(1-3), pp. 689-723 (1982).

20. Peskin, C.S. "The immersed boundary method", Acta Numerica, 11, pp. 479-517 (2002).

21. Zhu, L. and Peskin, C.S. "Simulation of a flapping flexible filament in a flowing soap film by the immersed boundary method", Journal of Computational Physics, 179(2), pp. 452-468 (2002).

22. Baaijens, F.P.T. "A fictitious domain/mortar element method for fluid-structure interaction", International Journal for Numerical Methods in Fluids, 35(7), pp. 743-761 (2001).

23. Swim, E.W. and Seshaiyer, P. "A nonconforming finite element method for fluid-structure interaction problems", Comput. Methods Appl. Mech. Eng., 195(1718), pp. 2088-2099 (2006).

24. Wang, S.Y. "A finite element model for the static and dynamic analysis of a piezoelectric bimorph", Int. J. Solids Struct., 41(15), pp. 4075-4096 (2004).

25. Namkoong, K., Choi, H.G. and Yoo, J.Y. "Computation of dynamic fluid-structure interaction in twodimensional laminar flows using combined formulation", J. Fluids Struct., 20(1), pp. 51-69 (2005).

26. Glück, M., Breuer, M., Durst, F., Halfmann, A. and Rank, E. "Computation of fluid-structure interaction 
on lightweight structures", Journal of Wind Engineering and Industrial Aerodynamics, 89(14-15), pp. 13511368 (2001).

\section{Biographies}

Yasser Amini received his BS, MS, and $\mathrm{PhD}$ degrees in Mechanical Engineering from Shiraz University, Iran, in 2006, 2009 and 2014, respectively. $\mathrm{He}$ is currently Associate Professor in the Department of Mechanical Engineering at the Persian Gulf University, Iran. His research interests include: CFD, mesh-less methods, SPH method, Fluid structure interaction, rarefied gas flow dynamics, smart materials and DSMC method.

Homayoun Emdad received his B.S. degree in Aerospace Engineering from Washington University,
USA, in 1982, and his MS and PhD degrees in the same subject from Kansas University, USA in 1984 and 1988, respectively. He is currently Associate Professor in the School of Mechanical Engineering at Shiraz University, Iran. His primary research interests are computational fluid dynamics, flow control, fluid structure interaction, mesh-less methods, multiphase flow, and aerodynamics.

Mehrdad Farid received BS and MS degrees in Mechanical Engineering from Shiraz University, Iran, in 1985 and 1988, respectively, and his $\mathrm{PhD}$ degree in the same subject from the University of Calgary, USA, in 1997. He is currently Associate Professor in the School of Mechanical Engineering at Shiraz University, Iran. His primary research interests are computational mechanics, vibration and control analysis, fluid structure interaction and smart materials. 